\title{
Barotropic Instability of a Cyclone Core at Kilometer\#Scale Resolution
}

\section{Journal Article}

\section{Author(s):}

Leutwyler, David (D); Schär, Christoph (i)

Publication date:

2019-11

Permanent link:

https://doi.org/10.3929/ethz-b-000373267

\section{Rights / license:}

Creative Commons Attribution 4.0 International

\section{Originally published in:}

Journal of Advances in Modeling Earth Systems 11(11), https://doi.org/10.1029/2019ms001847

\section{Funding acknowledgement:}

154486 - Cloud-resolving climate modeling on future supercomputing platform (SNF) 


\section{JMES Journal of Advances in Modeling Earth Systems}

\section{RESEARCH ARTICLE \\ 10.1029/2019MS001847 \\ Barotropic Instability of a Cyclone Core at Kilometer-Scale Resolution}

Key Points:

- Kilometer-scale near-global

simulations of idealized baroclinic

waves resolve secondary instability at a cold front

- Limited-area real-case simulation of an observed event confirms realism of the simulation

- Reduced deformation near cyclone core allows secondary barotropic instability to grow

Supporting Information:

- Supporting Information S1

Correspondence to:

D. Leutwyler,

david.leutwyler@mpimet.mpg.de

Citation:

Leutwyler, D., \& Schär, C. (2019).

Barotropic instability of a cyclone

core at kilometer-scale resolution.

Journal of Advances in Modeling Earth

Systems, 11, 3390-3402. https://doi.org/

10.1029/2019MS001847

Received 6 AUG 2019

Accepted 25 SEP 2019

Accepted article online 16 OCT 2019

Published online 6 NOV 2019

\author{
David Leutwyler ${ }^{(\mathbb{D})}$ and Christoph Schär ${ }^{1}$ (D) \\ ${ }^{1}$ Atmospheric and Climate Science, ETH Zürich, Zürich, Switzerland
}

\section{Introduction}

Abstract Secondary disturbances spawning frontal waves along the fronts of mature midlatitude low-pressure systems were identified decades ago from satellite images and during field campaigns. Today's flagship supercomputers allow performing simulations at kilometer-scale resolution on computational domains covering the entire lifecycle of synoptic-scale systems and thus enable explicit representation of small-scale disturbances embedded in large-scale circulations. Here we demonstrate these capabilities in two different types of kilometer-scale simulations. The first is a 10-day-long near-global simulation of an idealized moist baroclinic wave, performed at $1 \mathrm{~km}$ grid spacing and employing 16,001 ×36,006 $\times 60$ grid points. The second is a real-case simulation of an extratropical low-pressure system, driven by the European Centre for Medium-Range Weather Forecasts's operational analysis. At kilometer-scale resolution, both simulations display clear evidence of embedded mesoscale vortices spawning along frontal systems of mature extratropical cyclones. The vortices appearing in the real-case simulation can also be identified in satellite imagery of the system. The simulated developments are due to a barotropic instability mechanism and driven by strong low-level horizontal wind shear. While the simulation of the frontal systems is amenable at model resolutions around $10-50 \mathrm{~km}$, the instability mechanism itself relies on the representation of a narrow shear zone, requiring about 5 times finer resolution. Results suggest that the flow regimes suppressing or fostering barotropic vortices can coexist in the same synoptic system. Far away from the cyclone core, the instability is suppressed by deformation associated with the large-scale flow, while close to the mature cyclone core, the narrow frontal structure becomes unstable.

The midlatitude atmospheric dynamics are strongly affected by fluid-dynamical instabilities of barotropic and baroclinic type. The development of these concepts is closely tied to the history of synoptic meteorology. About 100 years ago, the polar front theory postulated that cyclones emerge when frontal-wave instabilities grow on narrow cold frontal surfaces (Bjerknes \& Solberg, 1922). The polar front theory quickly became the backbone of early meteorological forecasting. However, about 50 years later, numerical experiments revealed that fronts emerge when disturbances grow to finite amplitude in an initially smooth baroclinic environment (Edelmann, 1963; Hoskins \& West, 1979). As this theory was able to successfully explain the emergence of both cyclones and fronts in an initially smooth baroclinic zone, interest in frontal wave theories waned. However, synopticians continued to mark frontal waves on surface weather charts.

One of the early studies that resumed theoretical work on frontal waves was that of Moore and Peltier (1987). They argued that classical baroclinic instability would be unable to explain the small horizontal wavelengths seen in a series of frontal waves and proposed a new "cyclone-scale mode of baroclinic instability." Unlike the classical baroclinic instability, this mode was not amenable by the balanced dynamics but required sophisticated analysis using the hydrostatic primitive equations. However, later studies were unable to confirm the existence of this mode (Snyder, 1995).

Today frontal waves are interpreted as being related to a barotropic instability process. Early studies of this type isolated a balanced instability related to a low-level frontal potential vorticity (PV) anomaly (Joly \& Thorpe, 1990; Malardel et al., 1993) and/or to a low-level warm anomaly ahead of the cold front (Schär \& Davies, 1990). While the background states considered in these studies differ, the basic instability mechanisms are very similar. The energy source of the instability is the across-frontal wind shear (rather than the temperature contrast as in baroclinic instability), and the most unstable mode has a length scale of about 5-10 times the width of the front. (c)2019. The Authors.

This is an open access article under the terms of the Creative Commons Attribution License, which permits use, distribution and reproduction in any medium, provided the original work is properly cited. 


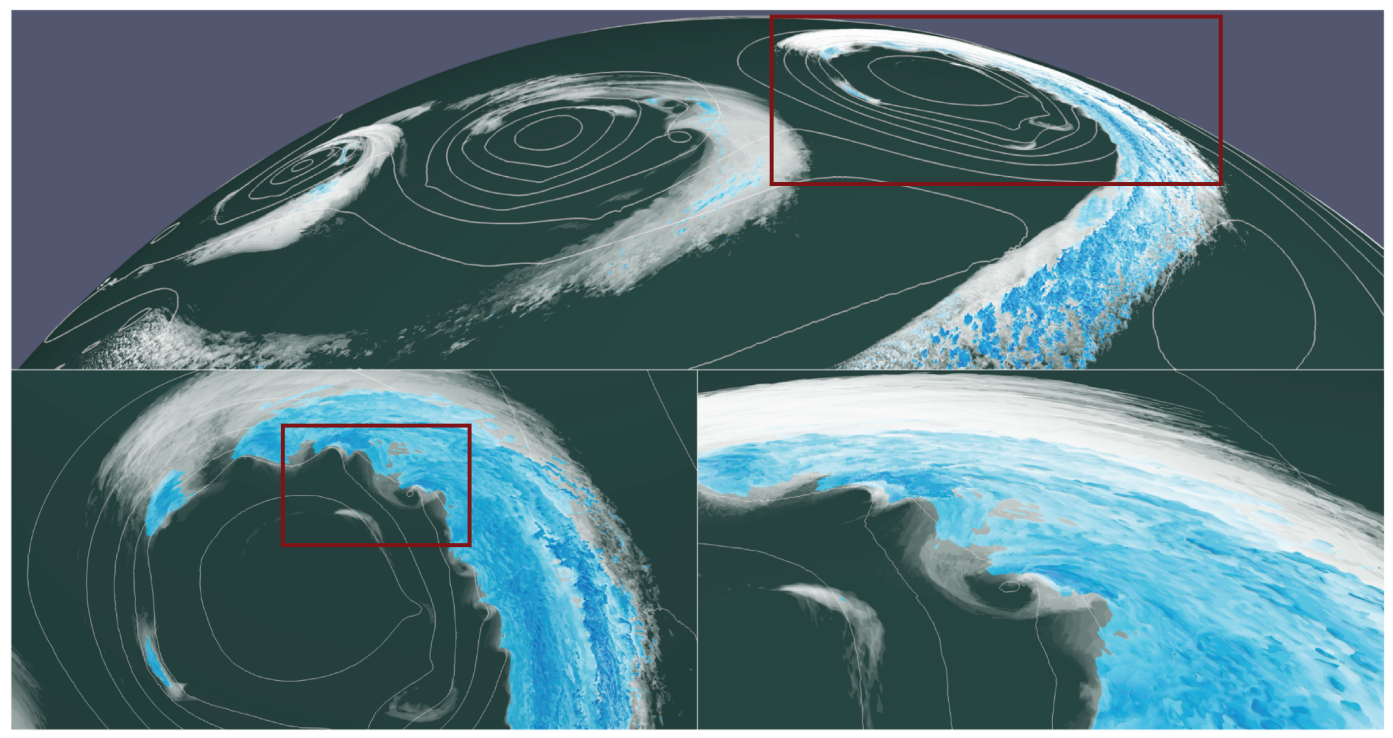

Figure 1. (top) Perspective view of an idealized moist baroclinic wave at Day 10 of a near-global simulation with $1 \mathrm{~km}$ grid spacing and (bottom) mesovortices embedded in the frontal system. White shading $=$ volume rendering of the sum of cloud ice, cloud water, and graupel $\geq 10^{-3} \mathrm{~g} / \mathrm{kg}$; blue shading = isosurface of rain and snow hydrometeors $\geq 4 \cdot 10^{-2} \mathrm{~g} / \mathrm{kg}$. This simulation has previously been presented by Fuhrer et al. (2018).

From a conceptual perspective, frontal-wave developments can be considered a two-stage process (Bishop \& Thorpe, 1994a, 1994b; Dacre \& Gray, 2006; Joly \& Thorpe, 1990): (i) Increased deformation strengthens an existing surface front, and upward motions with latent heat release enable the formation of an approximately two-dimensional low-level sheet of enhanced PV below the level of maximum condensational heating. The maximum of PV in this sheet implies that the criterion for barotropic instability is satisfied (Holton \& Hakim, 2013), but the flow deformation associated with frontogenesis may suppress the instability. (ii) As the cyclone matures, a reduction in deformation favors the instability and may lead to wave-like perturbations along the front, which may grow and break up into a series of vortices.

There is ample observational evidence for the relevance of barotropic mechanisms in synoptic-scale atmospheric dynamics. Although baroclinic developments are considered the mainstay of cyclogenesis, frontal waves are a commonly observed feature (e.g., Chaboureau \& Thorpe, 1999; Rivals et al., 1998). Recently Schemm and Sprenger (2015) estimated, based on the European Centre for Medium-Range Weather Forecasts (ECMWF) (ERA)-Interim Re-Analysis (Dee et al., 2011), that in the North Atlantic basin frontal-wave cyclogenesis accounts to about $10 \%$ of all deep cyclogenesis events. Note that frontal waves often remain of small amplitude, and their genesis may not be fully captured by the forecast model underlying the ERA-Interim reanalysis. Anyhow, some of the events are associated with rapid cyclogenesis and may lead to highly significant weather events, in particular, if moist dynamics and phase-locking between upper and lower-level wave anomalies are considered (e.g., Fehlmann \& Davies, 1999; Ludwig et al., 2015). Even frontal waves that remain rather weak may lead to substantial variations in weather phenomena (Joly et al., 1999).

The key challenge when forecasting frontal waves is related to the small horizontal scales. Unlike baroclinic instability, which emerges at well-resolved wavelengths of around 4,000 km and is determined by the depth of the troposphere (Holton \& Hakim, 2013), the most unstable modes of barotropic-type instabilities are governed by the width of the shear zone and can thus even challenge today's model resolutions. An instability with a wavelength of about $100 \mathrm{~km}$, for example, requires an effective resolution of about $10 \mathrm{~km}$ for the instability to emerge, which in turn requires a grid spacing of about $2 \mathrm{~km}$.

In the current study, we investigate an idealized and a real-case event of a barotropic instability in a cyclone core. The idealized case has previously been investigated in a benchmark simulation (see Figure 1), while the real-case simulations follow an observed case of cyclone core instability. In both cases, the instability derives from narrow cold frontal near-surface wind shears when the front is advected into the cyclone core and exhibits pronounced signs of rolling up. In doing so, we exploit a nonhydrostatic model with resolution 
around $1 \mathrm{~km}$. The added value of performing simulations at such high resolutions has so far predominantly been related to the improved representation of moist-convective processes. Explicitly representing moist convection leads to better representation of precipitation characteristics (Ban et al., 2014; Clark et al., 2016; Prein et al., 2015), more credible interactions with synoptic and mesoscale systems (Leutwyler et al., 2016), and convectively generated gravity waves in the tropics (Müller et al., 2018). Added value can also be attributed to the high-resolution surface fields used at the lower boundary condition. Their refined spatial detail yields an improved simulation of surface winds (Belušić et al., 2018), or more credible land-surface feedbacks (e.g., Hohenegger et al., 2008; Imamovic et al., 2017), and orographic and gravity-wave drag processes (Shutts \& Vosper, 2001; Vosper et al., 2016). Representation of barotropically unstable waves, on the other hand, portrays another phenomenon-emerging in the absence of orographic forcing. Although established theory draws from semigeostrophic dynamics, resolution is critical due to the small horizontal scales involved. As such, their representation in weather and climate models depicts yet another case for added value at kilometer-scale resolution.

The idealized simulations of the current study consider a near-global model domain and represent the evolution of a growing baroclinic wave to a mature cyclone, the subsequent formation and growth of barotropic wave trains on the narrow cold front, and ultimately the formation of mesovortices within the cyclone core. This sequence of events is facilitated by the use of a free-slip lower boundary condition, consistent with the setup of the idealized baroclinic wave (Jablonowski \& Williamson, 2006; Park et al., 2013), which enables large near-surface horizontal wind shears.

While qualitatively similar behavior has been noted in several observational studies of maritime cyclones (Fu et al., 2004; Ralph, 1996), the formation of mesoscale vortices in the cyclone's core appears to be a rather rare phenomenon, likely due to the role of real-world surface friction in reducing low-level shear. We thus supplement the idealized case with a simulation of an observed case, where satellite imagery revealed a beautiful sequence of vortices in the cyclone core.

We document the simulations and outline the diagnostics used for the analysis of frontal deformation in unbalanced flows in section 2. After that, we present the results of the idealized and real-case simulations in section 3 and finally conclude the study with a discussion in section 4 .

\section{Simulations and Diagnostics}

\subsection{Secondary Instability in an Idealized Moist Baroclinic Wave}

In the first set of simulations, near-global simulations are conducted of an idealized baroclinic wave using version 5.0 of the weather and climate model COSMO (Förstner \& Doms, 2004; Steppeler et al., 2003). They follow the configuration of Jablonowski and Williamson (2006) extended with an initial vertical moisture profile (Park et al., 2013). The simulations are initialized from a hydrostatically balanced baroclinic flow disturbed by a large-scale Gaussian perturbation along the dynamically unstable Northern Hemisphere jet stream.

The simulations have already been presented by Fuhrer et al. (2018) and were at first meant to assess the feasibility of multidecadal near-global kilometer-resolution climate simulations. The simulations were run on a dedicated supercomputer equipped with 4,888 nodes, each containing an accelerator based on graphics processing units. Due to the extremely high costs of this simulation, it has not been possible to rerun the simulation for the present paper, and the analyses presented here must thus rely on the model output written previously. While in Fuhrer et al. (2018) primarily validation for dry and moist configurations of the test case was performed for the early phase of development, here we investigate the later phase of the simulations when the secondary instability develops, in more detail.

The simulations employ a regular longitude-latitude mesh on a sphere. This configuration requires additional treatment near the poles since the distance between grid points becomes smaller and smaller as the pole is approached. To address this singularity, the computational domain only spans a latitude belt ranging from $80^{\circ}$ south to $80^{\circ}$ north in order to avoid numerical instabilities appearing as the mesh collapses. The solution is relaxed against the initial conditions at the northern and southern domain boundaries while using periodic boundary conditions in the east-west direction. The domain covers $98.5 \%$ of the Earth's surface area, but at kilometer-scale resolution, the meridional grid spacing nevertheless reduces to $174 \mathrm{~m}$ at the northern/southern boundaries. 
The idealized simulations were conducted for a 10-day-long period at three model resolutions: (i) at $10 \mathrm{~km}$ grid spacing (at the equator), using a time step of $60 \mathrm{~s}$ and employing 3,606 $\times 1,601 \times 60$ grid points; (ii) at $2 \mathrm{~km}$ grid spacing, using a time step of $12 \mathrm{~s}$ and employing $18,006 \times 8,001 \times 60$ grid points; and (iii) at $1 \mathrm{~km}$ grid spacing, employing $36,006 \times 16,001 \times 60$ grid points and a time step of $6 \mathrm{~s}$. Depending on the experiment, moist processes are parameterized using a single-moment bulk microphysics scheme (Reinhardt \& Seifert, 2006) that includes five hydrometeor species (cloud water, cloud ice, rain, snow, and graupel).

\subsection{Secondary Instability in an Extratropical Low-Pressure System}

In the second set of simulations, a limited-area high-resolution simulation is conducted of a real-case event using a two-step one-way nesting approach. The configuration is similar to what has been described in Leutwyler et al. (2016), but the computational domain is located over the Eastern Atlantic. On 22 February 2016 at 12 UTC, a coarse simulation with a grid spacing of $\Delta x \approx 12 \mathrm{~km}$ and employing $300 \times 260 \times 60$ grid points is initialized, based on initial and boundary conditions derived from ECMWF operational analysis, at the time employing a grid spacing of about $9 \mathrm{~km}$. Herein a second simulation is nested at hourly boundary update interval, using a refined horizontal grid spacing of $2.2 \mathrm{~km}$. Due to the fine resolution, deep convection is treated explicitly by the governing equations. The numerical mesh for the high-resolution simulation employs $1,542 \times 800 \times 60$ grid points, large enough to cover the maturing phase of the low-pressure system. The simulation period covers its occluding phase, and analysis is provided after $27 \mathrm{hr}$ of model integration.

\subsection{Frontogenesis and Deformation}

As outlined above, the growth of barotropic instabilities relates to classical theory of frontogenesis in adiabatic flows and especially to the evolution of frontal deformation. Here we provide a summary of how a diagnostic of frontal deformation can be obtained. While the derivation closely follows a previous publication (Schär \& Wernli, 1993), we here consider frontogenesis in an unbalanced flow.

The starting point is the thermodynamic equation in the form

$$
\frac{\partial \theta}{\partial t}+\vec{u} \cdot \nabla \theta=0
$$

Here $\vec{u}$ is the three-dimensional velocity vector and $\theta$ the potential temperature. Under certain conditions the vertical motion can be neglected. We assume that this is a good approximation at low levels over oceans, when the flow has to follow the lower boundary and when diabatic perturbations of (1) are small. In such cases

$$
\frac{\partial \theta}{\partial t}+\vec{v} \cdot \nabla_{h} \theta=0
$$

where $\vec{v}=(u, v)$ denotes the horizontal wind. Following Keyser et al. (1988), we differentiate (2) with respect to $x$ to yield

$$
\frac{\partial \theta_{x}}{\partial t}+\vec{v} \cdot \nabla_{h} \frac{\partial \theta}{\partial x}+\frac{\partial \vec{v}}{\partial x} \cdot \nabla_{h} \theta=0
$$

or, after rewriting the first and second terms on the left-hand side and rearranging,

$$
\frac{D_{h} \theta_{x}}{D t}=-\frac{\partial \vec{v}}{\partial x} \cdot \nabla_{h} \theta=-u_{x} \theta_{x}-v_{x} \theta_{y}
$$

A similar equation is obtained for the $y$ direction. Combining these two equations yields

$$
\frac{D_{h} \nabla_{h} \theta}{D t}=-\left(\begin{array}{l}
u_{x} \theta_{x}+v_{x} \theta_{y} \\
u_{y} \theta_{x}+v_{y} \theta_{y}
\end{array}\right)=\vec{Q} .
$$

The vector $\vec{Q}$ is closely related to the $Q$ vector of the quasi-geostrophic dynamics (Hoskins et al., 1978), but here it is used with the full horizontal wind (rather than the geostrophic flow). Equation (3) may be cast into its matrix form

$$
\frac{D_{h} \nabla_{h} \theta}{D t}=\vec{Q}=\mathbf{Q} \nabla_{h} \theta \text { with } \mathbf{Q}=-\left[\begin{array}{ll}
u_{x} & v_{x} \\
u_{y} & v_{y}
\end{array}\right]
$$

Here the matrix $\mathbf{Q}$ is only dependent upon the local kinematic properties of the flow field and expresses a mapping from the potential temperature gradient $\nabla_{h} \theta$ to its total rate of change $D_{h} \nabla_{h} \theta / D t$. The nature of frontolytical and frontogenetical effects can hence be analyzed by studying the properties of $\mathbf{Q}$. 
For this purpose, it is convenient to split Q, following Schär and Wernli (1993), as

$$
\mathbf{Q}=-\left[\begin{array}{ll}
u_{x} & v_{x} \\
u_{y} & v_{y}
\end{array}\right]=-\frac{D}{2}\left[\begin{array}{ll}
1 & 0 \\
0 & 1
\end{array}\right]+\frac{\xi}{2}\left[\begin{array}{rr}
0 & -1 \\
1 & 0
\end{array}\right]-\frac{1}{2}\left[\begin{array}{rr}
\alpha_{2} & \alpha_{1} \\
\alpha_{1} & -\alpha_{2}
\end{array}\right]=\mathbf{Q}_{\mathrm{div}}+\mathbf{Q}_{\mathrm{rot}}+\mathbf{Q}_{\mathrm{def}}
$$

where the three terms relate to the effects of divergence $\left(\mathbf{Q}_{\operatorname{div}}\right.$ with $\left.D=u_{x}+v_{y}\right)$, rotation $\left(\mathbf{Q}_{\text {rot }}\right.$ with $\xi=$ $\left.-u_{y}+v_{x}\right)$ and deformation $\left(\mathbf{Q}_{\text {def }}\right.$ with $\alpha_{1}=u_{y}+v_{x}$ and $\left.\alpha_{2}=u_{x}-v_{y}\right)$, respectively.

\subsubsection{Deformation}

The deformation matrix $\mathbf{Q}_{\text {def }}$, that is, the third term in equation (5), has the eigenvalues

$$
\lambda_{ \pm}= \pm \frac{1}{2} \alpha \quad \text { with } \quad \alpha=\left(\alpha_{1}+\alpha_{2}\right)^{\frac{1}{2}}
$$

and the eigenvectors

$$
\vec{e}_{ \pm}= \begin{cases}\frac{1}{\sqrt{2} \alpha}\left(\begin{array}{l}
-\alpha \\
\pm \alpha
\end{array}\right) & \left(\text { if } \alpha_{2}=0\right) \\
\frac{1}{2 \sqrt{\alpha \mp \alpha_{1}}}\left(\begin{array}{l} 
\pm \alpha-\alpha_{1}-\alpha_{2} \\
\pm \alpha-\alpha_{1}+\alpha_{2}
\end{array}\right) & \text { if } \left.\alpha_{2} \neq 0\right)\end{cases}
$$

The eigenvectors are orthogonal, $\lambda_{ \pm}$denotes the strength of the deformation flow, and $\vec{e}_{+} / \vec{e}_{-}$point into the directions of frontogenesis and frontolysis, respectively.

The formulation provided above is in principle directly applicable to an unbalanced flow. However, on a numerical level it might be difficult to properly distinguish between the divergent and deformational parts of the kinematics. To ensure a proper separation, let us assume that the nondivergent part of the flow is described by a stream function, that is,

$$
u=-\psi_{y} \text { and } v=\psi_{x}
$$

The so-defined wind field has the property that it is divergence free, that is, $D=0$, and the vorticity can be obtained as

$$
\xi=-u_{y}+v_{x}=\psi_{x x}+\psi_{y y}=\Delta \psi
$$

where $\Delta$ denotes the Laplacian.

As the divergence matrix disappears by design, the deformation matrix can easily be computed as

$$
\mathbf{Q}_{\mathrm{def}}=\mathbf{Q}-\mathbf{Q}_{\mathrm{rot}}=-\left(\begin{array}{ll}
-\psi_{x y} & \psi_{x x} \\
-\psi_{y y} & \psi_{x y}
\end{array}\right)-\frac{\Delta \psi}{2}\left(\begin{array}{cc}
0 & -1 \\
1 & 0
\end{array}\right)
$$

and thus

$$
\mathbf{Q}_{\mathrm{def}}=\frac{1}{2}\left(\begin{array}{cc}
2 \psi_{x y} & -\psi_{x x}+\psi_{y y} \\
-\psi_{x x}+\psi_{y y} & -2 \psi_{x y}
\end{array}\right)
$$

Comparison with (5) then yields

$$
\alpha_{1}=\psi_{x x}-\psi_{y y} \quad \text { and } \quad \alpha_{2}=-2 \psi_{x y}
$$

\subsubsection{Implementation}

In unbalanced flows, the divergence-free stream function (8) can be obtained by employing a Helmholz-Hodge Decomposition of the low-level flow field. It relies on the fundamental theorem of vector calculus (Helmholtz's theorem) which states that a sufficiently smooth vector field can be represented as the sum of a rotating nondivergent $(D=0)$ and a divergent nonrotating $(\xi=0)$ part. To this end, we make use of the methodology outlined in Bhatia et al. (2014), which allows the decomposition of a bounded vector field.

For the simulation of the baroclinic wave (see section 3.1), the resulting stream function appears overall smooth, but some small artifacts remain close to the lateral boundaries. Since the distance of the boundaries to the domain of interest remains large $\left(>7^{\circ}\right)$, we consider the procedure good enough for our purpose. 

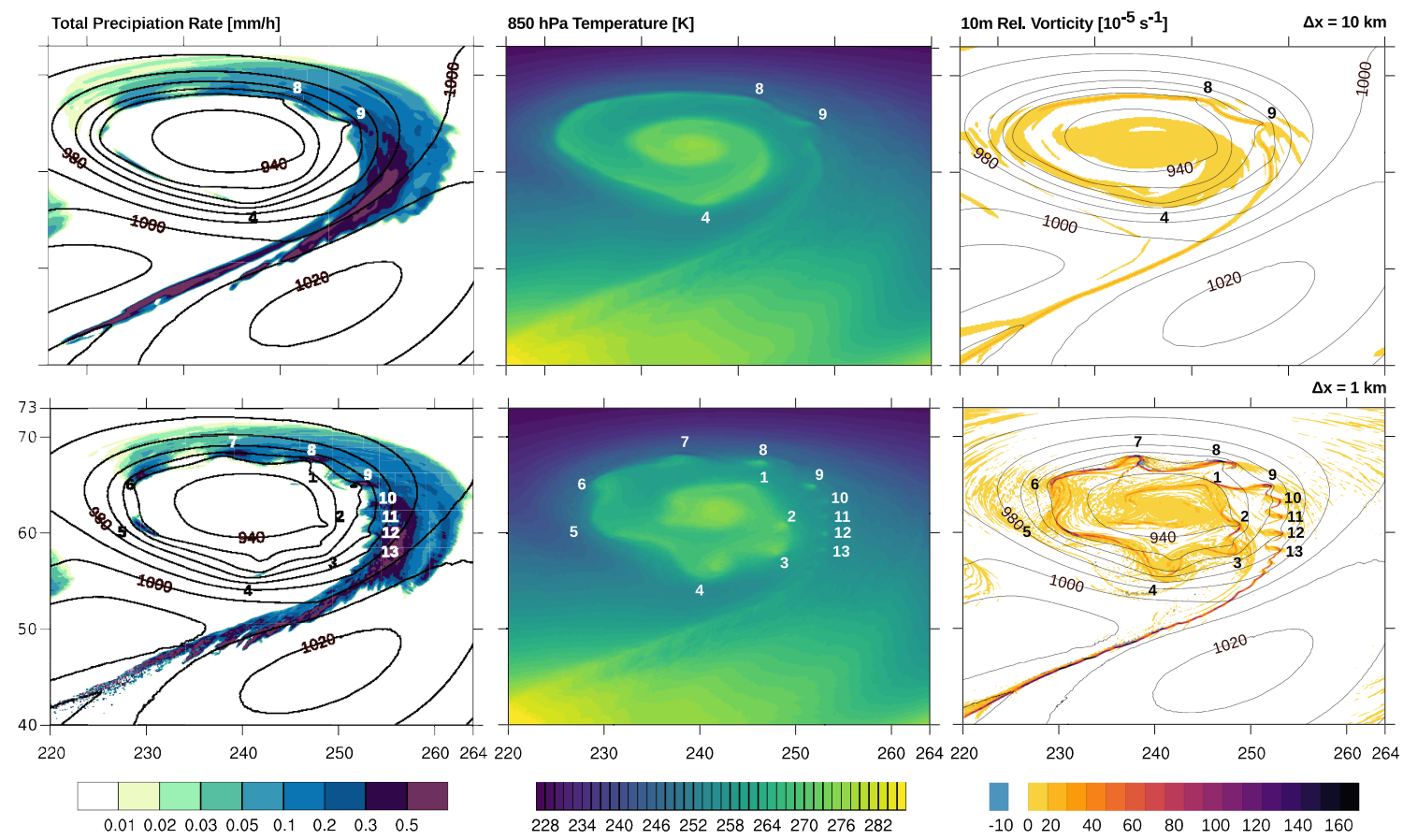

Figure 2. Zoomed details of idealized simulations. Vortices embedded in the cold front of the low-pressure system, labeled from 1-13. Representation in a simulation with (top) $10 \mathrm{~km}$, and (bottom) $1 \mathrm{~km}$ grid spacing. (left) Precipitation rate (mm/h), (middle) temperature on the $850 \mathrm{hPa}$ pressure level, and (right) relative vorticity at $10 \mathrm{~m}$ height. The black isolines denote surface pressure, with a line spacing of $10 \mathrm{hPa}$. In the bottom panel, the numbers label mesovortices, identified by eye. In the top panel the location of Mesovortices 4, 8, and 9 are marked accordingly. For display purposes, negative values of vorticity have been clipped at $-10^{-5} \mathrm{~s}^{-1}$. Typical vorticity values along the narrow frontal vorticity sheet amount to $60 \cdot 10^{-5} \mathrm{~s}^{-1}$, but occasionally values of up to $160 \cdot 10^{-5} \mathrm{~s}^{-1}$ can be found. Note that, for better perceptibly, the vorticity field displayed in the bottom right panel has been aggregated following the methodology outlined in section 2.3.3. A version on the original grid is provided in supporting information Figure S2, and an enhanced zoom of this figure is presented in supporting information Figure S1.

Once the stream function is known on the computational mesh, we employ finite differences for the computation of the eigenvectors and eigenvalues of the deformation matrix. Assuming that $\psi=\psi_{i, j}$ is available on a regular grid with grid spacings $\Delta x$ and $\Delta y$, and upon using centered finite differences in space, one obtains

$$
\begin{gathered}
\left(\psi_{x x}\right)_{i, j}=\frac{1}{\Delta x^{2}}\left[\psi_{i-1, j}-2 \psi_{i, j}+\psi_{i+1, j}\right] \\
\left(\psi_{y y}\right)_{i, j}=\frac{1}{\Delta y^{2}}\left[\psi_{i, j-1}-2 \psi_{i, j}+\psi_{i, j+1}\right] \\
\left(\psi_{x y}\right)_{i, j}=\frac{1}{4 \Delta x \Delta y}\left[\psi_{i+1, j+1}-\psi_{i+1, j-1}-\psi_{i-1, j+1}+\psi_{i-1, j-1}\right]
\end{gathered}
$$

Note that by design $\psi_{i, j}$ cancels out in (14). To obtain the eigenvalues and eigenvectors, these quantities are used to compute $\alpha_{1,2}$ using (11), and finally the latter terms are substituted into (6) and (7).

The results are plotted in the relevant figure in terms of $\lambda_{+} \vec{e}_{+}$. As the sign of the eigenvector is arbitrary, both $+\lambda_{+} \vec{e}_{+}$and $-\lambda_{+} \vec{e}_{+}$are displayed, starting at the grid points considered. Overall the resulting deformation field is very close to the field obtained when considering the full unbalanced flow (rather than its representation with $\psi$ ), supporting the assumptions made in (2).

\subsubsection{Visualization}

At the time of writing, consumer printers and screens yield an inadequate representation of the involved small-scale structures. The problem arises from the simulations of the 1 and $2 \mathrm{~km}$ simulations containing more grid points than the maximum number of pixels supported by the current generation of devices and graphics rendering engines. To address the issue, the deformation and vorticity fields in Figures 2-4 were 


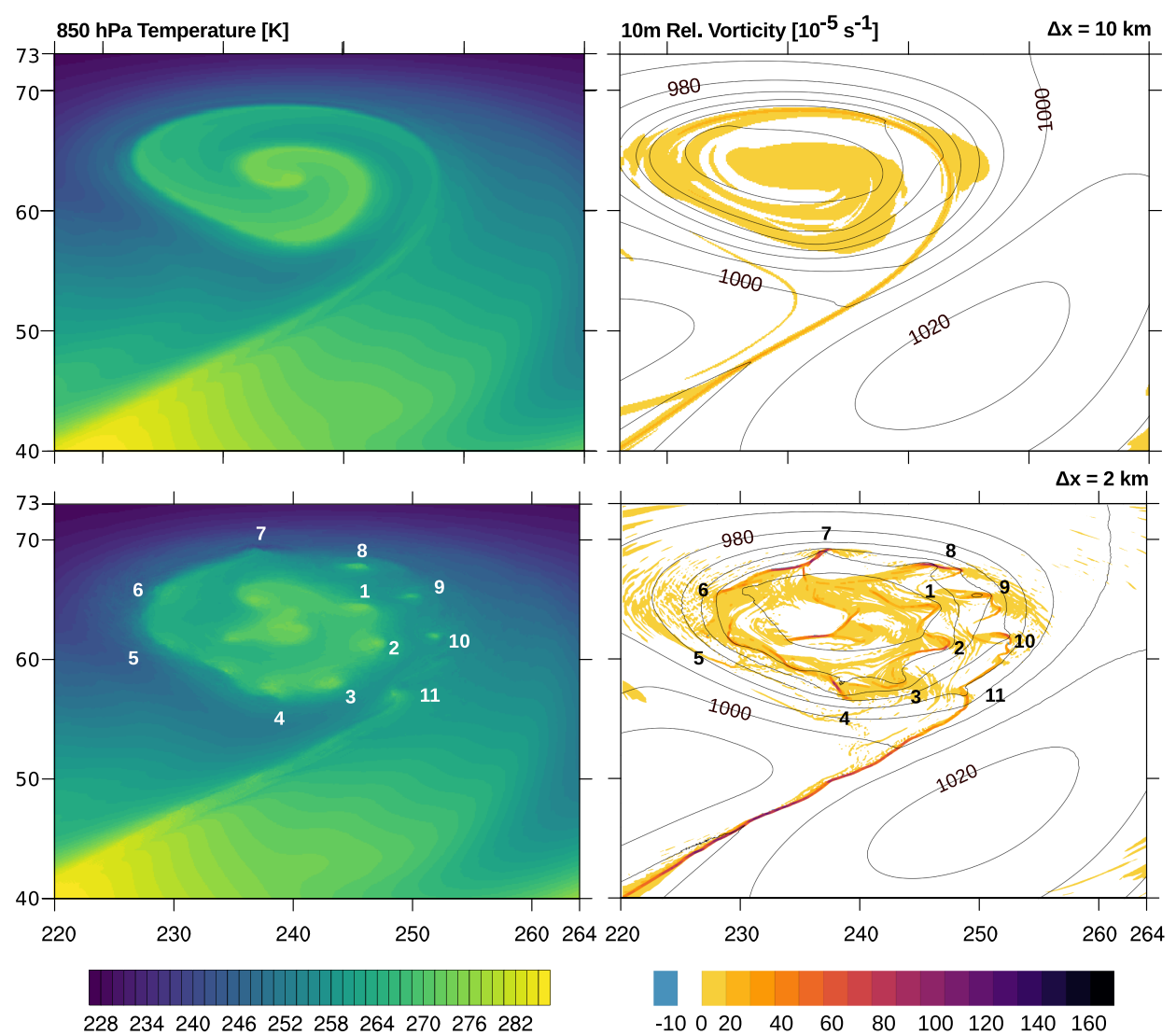

Figure 3. Temperature at $850 \mathrm{hPa}$ and vorticity as displayed in Figure 2, but for dry simulations conducted at 10 and $2 \mathrm{~km}$ grid spacing, respectively (top and bottom panels). Note that, similar to Figure 2, the vorticity field displayed in the bottom right panel has been aggregated following the methodology outlined in section 2.3.3. A version on the original grid is provided in supporting information Figure S2.

coarse grained. Since the presented analysis relies on local extrema, we aggregate the fields such that local maxima and minima are preserved:

$$
\tilde{\chi}(i, j)=\left\{\begin{array}{l}
\max _{\substack{n \\
\max _{k}=-n}} \chi(i+k, j+l), \text { if } \sum_{k, l=-n}^{n}(i+k, j+l)>0 \\
n \\
\min _{k, l=-n} \chi(i+k, j+l), \text { if } \sum_{k, l=-n}^{n}(i+k, j+l)<0
\end{array}\right.
$$

Here $\chi$ represents the original field, and $\tilde{\chi}$ the aggregated field. The calibration parameter $n$ denotes the width in model grid points considered for calculating the local extrema. It is set to a values of 0,3 , and 6 for the 10,2 , and $1 \mathrm{~km}$ simulations, respectively.

\section{Results}

\subsection{Secondary Instability in an Idealized Moist Baroclinic Wave}

In Fuhrer et al. (2018), we have presented the evolution of the growing baroclinic wave within an unstable westerly flow. As in the original studies (Jablonowski \& Williamson, 2006; Park et al., 2013), the development includes the growth of two low- and high-pressure systems after 4 days of model integration, and a third system appearing at Day 8. The systems undergo rapid deepening and transition into a nonlinear regime, accompanied by the formation of sharp precipitating fronts. Ultimately, the cyclonic low-pressure systems develop into deep cutoff warm-core vortices. After 10 days of integration, the cold/occluded front is advected into the core, exhibits signs of wrap-up, and breaking of the baroclinic wave is evident (Figure 1, top panel). 

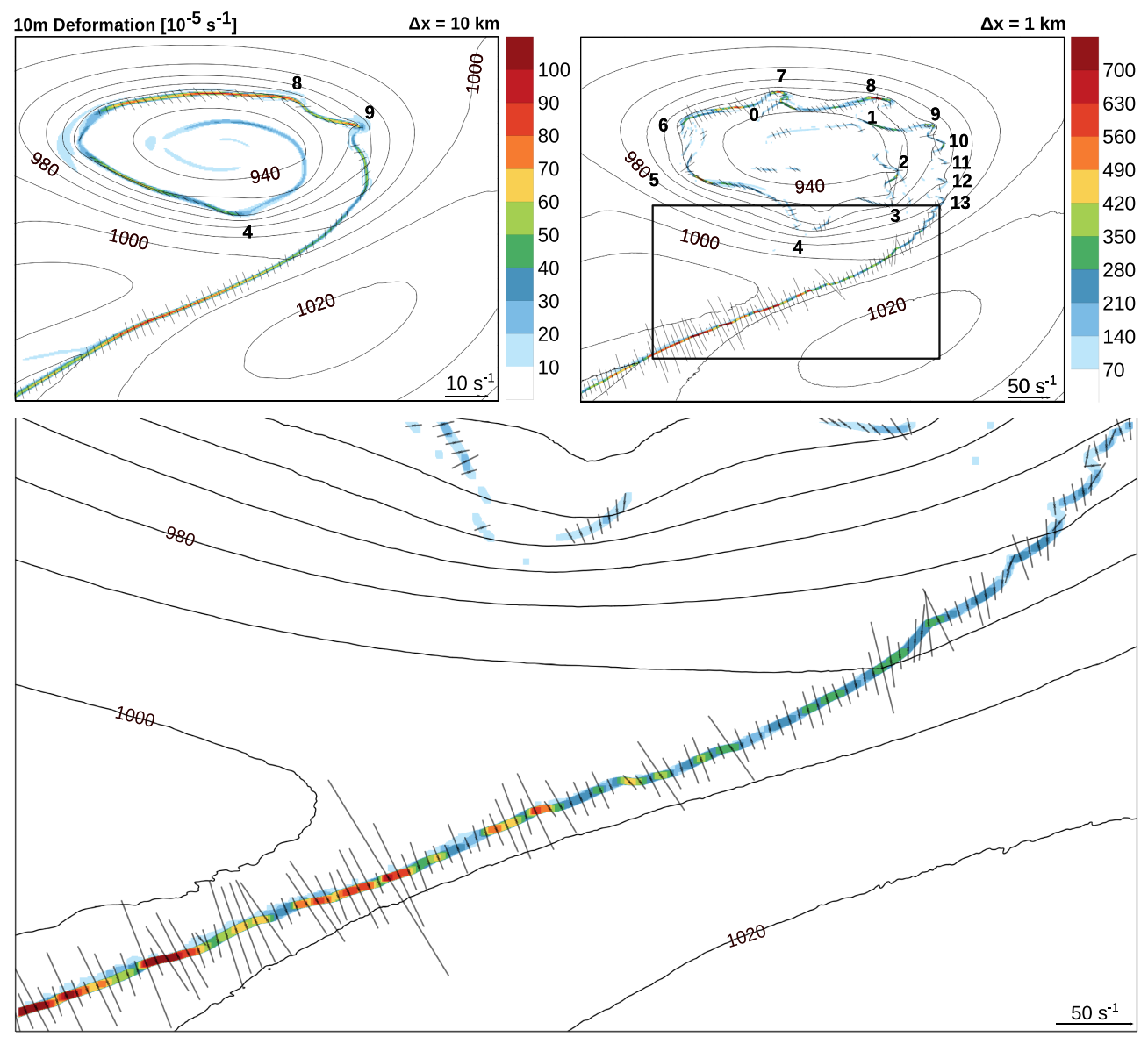

Figure 4. Deformation along the front of a moist barotropic wave in simulations performed at (top left panel) $10 \mathrm{~km}$ (top right and bottom panels) $1 \mathrm{~km}$ grid spacing. The black isolines denote surface pressure, with a line spacing of 10 $\mathrm{hPa}$; the numbers label mesovortices identified by eye (1-13), and the gray lines represent deformation vectors pointing in the direction of frontogenesis. The color shading displays the amplitude of the deformation (eigenvalue $\lambda_{+}$). Deformation vectors are plotted every $60 \mathrm{~km}$ in the top panels, and every $70 \mathrm{~km}$ in the bottom panel. Deformation vectors of less than $1 \cdot 10^{-5} \mathrm{~s}^{-1}$ are suppressed in the top left panel and $5 \cdot 10^{-5} \mathrm{~s}^{-1}$ in the top right and bottom panels. The black rectangle denotes the zoomed area displayed in the bottom panel. Note that, for better perceptibly, the deformation field of the $1 \mathrm{~km}$ simulations have been aggregated following the methodology outlined in section 2.3.3. A version on the original grid is provided in supporting information Figure S2, and an enhanced zoom of this figure is presented in supporting information Figure S1. For the derivation of deformation see section 2.3.

In the later stages of their development, the onset of a secondary instability can be observed in the clouds along the cold fronts of the two major systems (Figure 1, bottom panels). The instability manifests itself by destabilizing the trailing cold front (Figure 2 and supporting information Figure S1). While at $10 \mathrm{~km}$ grid spacing the instability leads to an undulating shape of the front, the perturbation is much more evident in the $1 \mathrm{~km}$ simulation. Here, wave-like disturbances propagate toward the core of the low-pressure system. Close to the surface, the $1 \mathrm{~km}$ simulation additionally exhibits warm-core mesovortices, forming a string of distinct temperature anomalies (Figure 2, middle panels). While mesovortices numbered 6-8 are about 200-300 km spaced apart, the distance increases up to $1,000 \mathrm{~km}$ (between mesovortices 4 and 5), as they are advected toward the cyclone core. Many of the developing mesovortices possess precipitating cloud systems (Figure 1, bottom panels, and Figure 2, left panels).

When performing these simulations without moist processes, a similar development results at kilometer-scale resolution, while the barotropic instability processes are not evident in a dry low-resolution $10 \mathrm{~km}$ simulation (see Figure 3). This result demonstrates that the formation of the low-level vorticity and PV sheet emerges from frontogenesis even in absence of moist dynamics. 

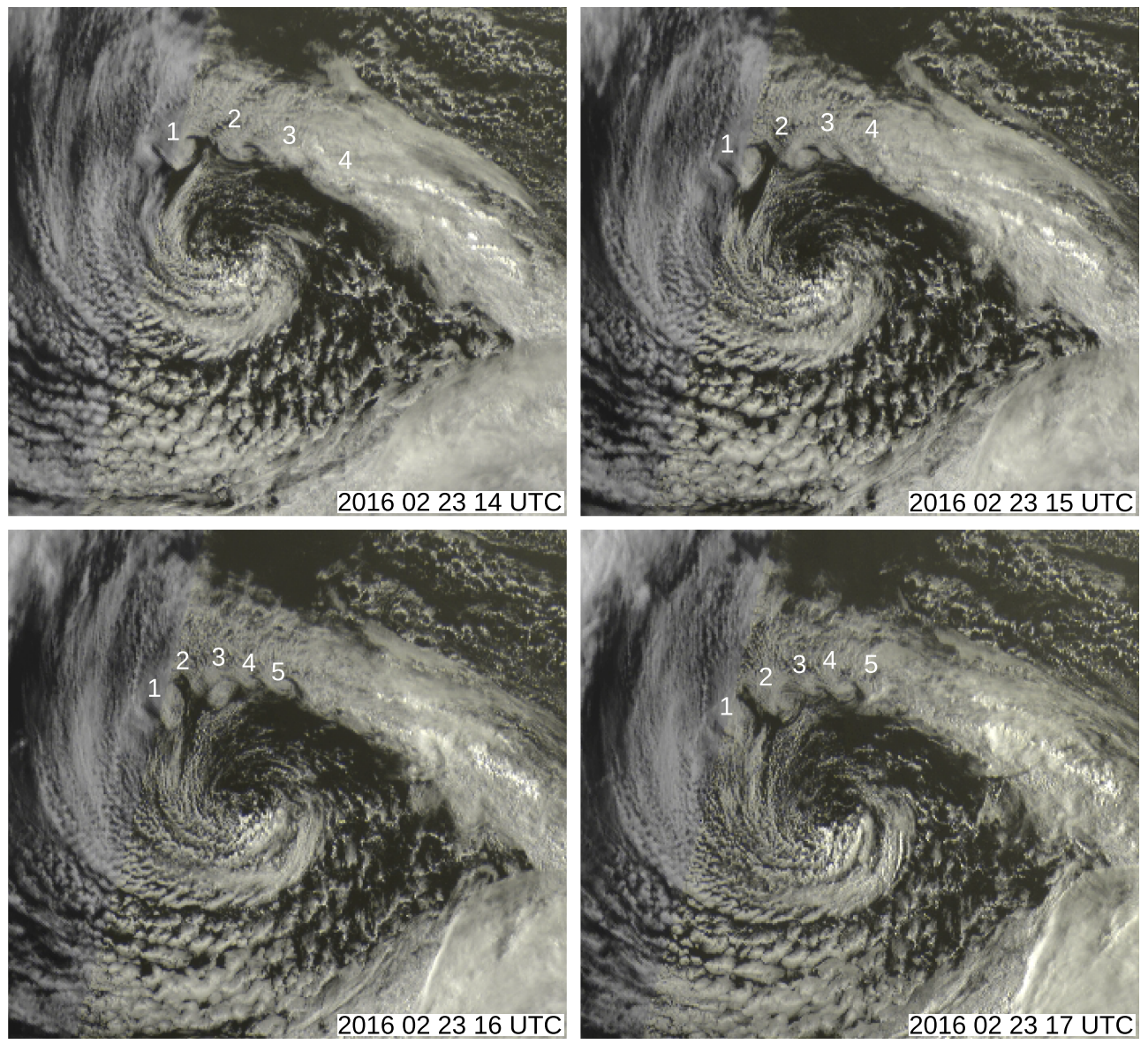

Figure 5. Snapshots from four SEVIRI/METEOSAT-10 satellite images of an extratropical low-pressure system, composed from the solar HRV channel. In each snapshot, the development of the low-level mesovortices forming along a zone of barotropic instability are labeled 1-5. All panels in this figure are based on RGB images obtained from Deutscher Wetterdienst (DWD).

\subsection{Along-Frontal Variations in Deformation}

The simulated evolution of wave-like structures along the cold front is consistent with the occurrence of a barotropic instability due to the presence of a low-level sheet of positive PV. As discussed in the introduction, the literature sees the development of frontal waves as a two-stage process. Most of the previous studies follow this interpretation. A first set of studies (e.g., Joly \& Thorpe, 1990) assumed a two-dimensional geometry with a preexisting low-level band of PV in absence of frontogenesis and deformation and addressed the evolution of the instability. Later studies (e.g., Bishop \& Thorpe, 1994a) have considered a time-dependent evolution of across-frontal deformation, thereby allowing the deformation to suppress the instability during an initial phase of development.

In the three-dimensional simulation presented in Figure 2, one can observe that both phases co-occur along different sections of the elongated cold front. Along the southwestern portion of the front, there is no indication of instability, due to the presence of frontogenesis and deformation in that section of the cold front. Here strong deformation suppresses the instability (Figure 4). As the front is advected toward and spirals around the center of the cyclone, active frontogenesis appears to cease (reduction of deformation by an order of magnitude), and the instability emerges. In other words, to the southwest of the system, the environmental flow squeezes the developing front, such that small undulations are immediately flattened out. Closer to the cyclone core, the deformation is less prominent, and thus, the instability can grow. Consistent with this interpretation, the growth in amplitude of the wave-like perturbations is accompanied by a growth in wavelength from initially $200-300 \mathrm{~km}$ to about $1,000 \mathrm{~km}$. This growth in wavelength is accompanied by a widening of the front as the vortices are advected toward the core of the system (Figure 2). Note also the 

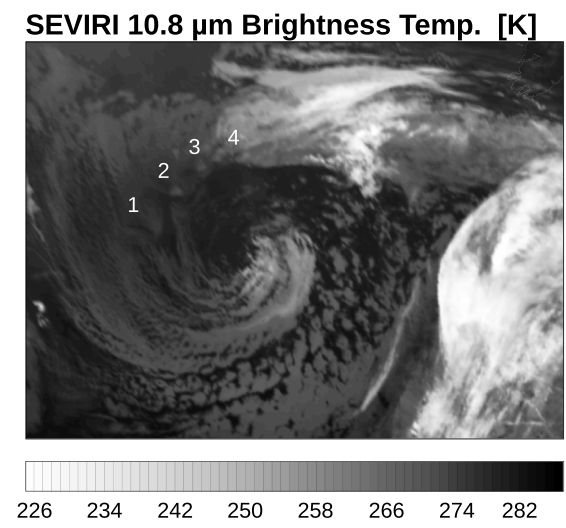

Windspeed [m/s] on $950 \mathrm{hPa}$ Level
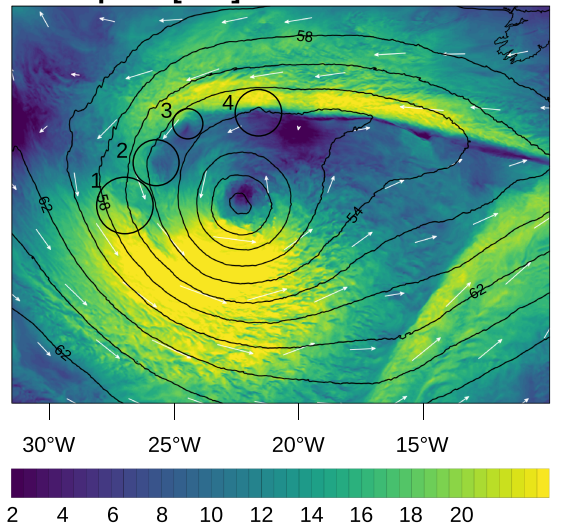
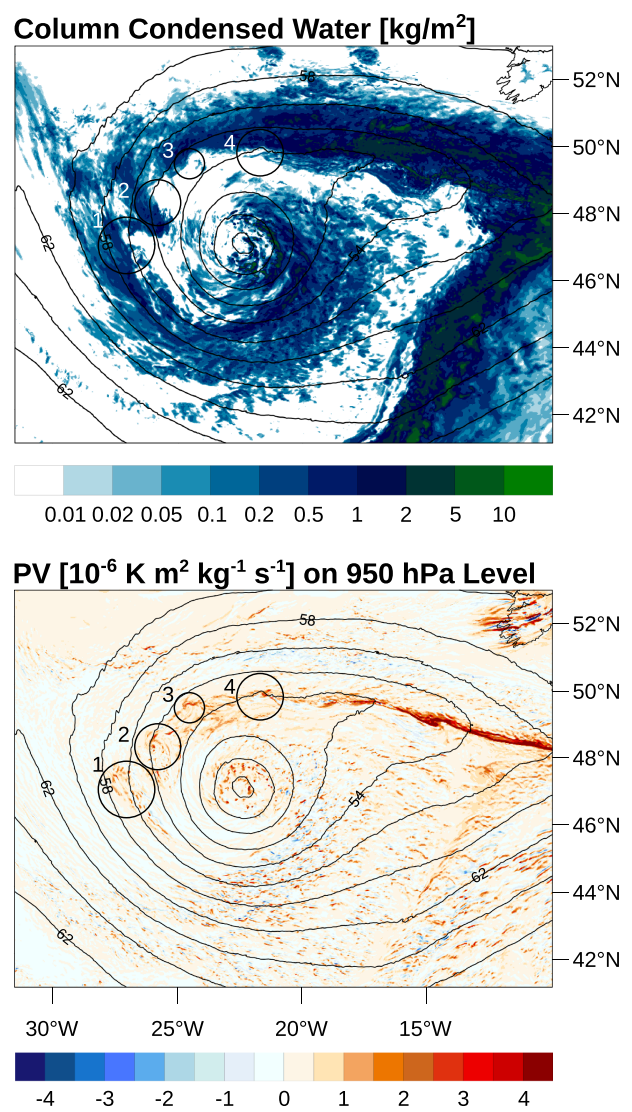

Figure 6. Mesovortices embedded in the front (labeled 1-4) of an occluding low-pressure system northwest of Portugal on 23 February 201615 UTC. (top left) Brightness temperature derived from the SEVIRI/METEOSAT-10 IR108 channel. The three remaining panels are based on a simulation with $2 \mathrm{~km}$ grid spacing, and each contains black contours denoting geopotential height on the $950 \mathrm{hPa}$ pressure level (gpdm). (top right) The blueish shading denotes vertically integrated column condensed water $\left(\mathrm{kg} / \mathrm{m}^{2}\right)$. (bottom left) The colored shading denotes horizontal wind speed on the $950 \mathrm{hPa}$ pressure level, and the white arrows wind vectors. (bottom right) The colored shading denotes potential vorticity (in PV units of $10^{-6} \mathrm{~K} \mathrm{~m}^{2} \cdot \mathrm{kg}^{-1} \cdot \mathrm{s}^{-1}$ ).

narrow structure of the deformation zone, illustrating the presence of an impressive scale collapse which is also evident in terms of vorticity.

Secondary instability along frontal systems is not only limited to idealized simulations. In the next section, we present a simulation of mesovortices appearing in an observed event over the North Atlantic.

\subsection{Secondary Instability in an Extratropical Low-Pressure System}

Around midnight of 20 February 2016, a synoptic-scale low-pressure system formed to the east of the North American continent, on the downstream side of an upper-level trough. During the next 2 days, it traversed across the Atlantic, reaching its deepest core pressure of about $1,000 \mathrm{hPa}$ (according to ECMWF analysis) at about $47.5^{\circ} \mathrm{N} / 33^{\circ} \mathrm{W}$ in the evening of 22 February 2016 . Over the next 2 days, the system slowly occluded and developed a comma-shaped cloud structure, while the core pressure rose to 1,010 hPa. Finally, on 24 February 2016 around 16 UTC, the system's core arrived at the Portuguese coast where it decayed. See the weather maps provided in supporting information Figure S4 for an overview of the large-scale environment.

During the occlusion phase of the low-pressure system, satellite images reveal a string of cyclonic mesovortices forming along the occluding front, located to the north of the cyclone core (Figure 5). They are rather uniformly spaced about 200-300 km apart and move coherently around the system core. The mesovortices can also be observed in infrared images (Figure 6, upper left panel). However, in infrared channels, mesovortices are usually more difficult to identify when they appear close to the surface, as long-wave radiances are more sensitive to upper-level clouds. 
In our kilometer-scale simulation of the same low-pressure system, small-scale waves (wavelength of about $100 \mathrm{~km}$ ) appear along the occluded section of the front, to the southwest of a high wind speed/shear region (Figure 6 and supporting information Figure S5). Over the next $12 \mathrm{hrs,} \mathrm{the} \mathrm{waves} \mathrm{develop} \mathrm{into} \mathrm{larger} \mathrm{dis-}$ turbances and finally into mesovortices spaced $200-300 \mathrm{~km}$ apart. As expected, the associated low-level PV sheet breaks up and forms cyclonic signatures (Figure 6, bottom right panel), also visible in the wind speed (Figure 6, bottom left panel). As in the idealized case discussed above, Figure S5 suggests that the instability grows along a narrow frontal feature and develops further as individual wave trains are advected toward the cyclone core. It is worth pointing out that the generation of PV in the current case is dominated by mechanisms acting along the warm front ahead of being ingested into the occluded front, rather than by mechanisms acting along the cold front (supporting information Figure S5). This constitutes a significant difference in comparison to the idealized case considered above, where vorticity and PV are generated along the cold front (Figure 2). As in the idealized simulation, the corresponding $12 \mathrm{~km}$ simulation displays slight undulations of the low-level PV sheet (see supporting information Figure S6).

\section{Discussion and Conclusions}

In idealized and real-study simulations at kilometer-scale resolution, we detected a secondary barotropic instability mechanism leading to mesovortices forming along fronts of occluding extratropical low-pressure systems: First we considered the simulation of an idealized moist baroclinic wave, following a moist version of the configuration by Jablonowski and Williamson (2006). Second, we considered a case study of an extratropical low-pressure system located to the west of Europe, displaying similar mesovortices as identified from satellite images. Simulations of the same cases, performed at a lower grid spacing of about $10 \mathrm{~km}$, displayed much weaker instability and no mesovortex formation. These results directly relate to studies established in the 1990s employing linear instability analyses. They suggest that a proportional relation exists between the wavelengths (the distance between two mesovortices), their growth rates, and the width of a low-level PV sheet. At that time, hypotheses were investigated by prescribing a two-dimensional low-level PV sheet, and hence, a gap remained, which requires demonstrating how such highly idealized numerical experiments relate to observed systems. Today's supercomputers enable conducting continental-scale to global simulations at kilometer resolution, now allowing testing and refining theory in more complex simulations.

The presented simulations demonstrate that the theories of frontal waves associated with barotropic-type instabilities (Bishop \& Thorpe, 1994a; Dacre \& Gray, 2006; Joly \& Thorpe, 1990; Schär \& Davies, 1990) are consistent with high-resolution fully three-dimensional simulations of idealized and real-case events of instabilities in a cyclone core. The analyses suggest that small-scale perturbations along sharp frontal features may develop into finite-amplitude mesoscale vortices. Consistent with theory, the wavelength of the mesoscale disturbances increases as the cyclone core is approached, and the PV sheet (which drives the instability) widens toward the core of the cyclone. Using the kilometer-resolution simulations presented, one can diagnose how the spatial structure of the developing cyclone appears relevant for the evolution of the instability process. Indeed, variations along the frontal structure-such as due to synoptic-scale deformation-may initially suppress instability along the fronts, before the instability can develop into frontal-wave like structures that disrupt the core of the cyclone.

Apparently, kilometer-scale resolution denotes a necessary condition for representing the width of the underlying PV sheet accurately enough. The hypothesis suggesting that condensational heating contributes to the formation of sharp frontal PV bands (Bishop \& Thorpe, 1994a; Ludwig et al., 2015) can be confirmed for our moist simulations, but it is worth pointing out that PV sheets also develop and become unstable in dry kilometer-resolution simulations. However, at lower resolution (10 km grid spacing), the conditions leading to the emergence of barotropic instability processes appear sensitive to moist processes. These results indicate that the presence of a pronounced scale collapse, which also occurs in dry frontogenesis, is in principle sufficient in generating low-level bands of PV, which can develop further into frontal instabilities. However, likely the diabatic generation of PV will dominate in most real-case settings, as a scale collapse will always invoke pronounced vertical lifting and likely trigger condensation-at least in cases of maritime cyclogenesis.

On a more practical note, the presented results impose a constraint on the Jablonowski and Williamson (2006) test, which constitutes an instrumental part of the standard procedure to test global atmospheric models. A compelling step of this validation consists of a grid refinement experiment, requiring convergence 
of the solution within the uncertainty of a high-resolution reference ensemble. It appears that at least for $\beta$ and $f$-plane variants of the test, solutions converge around a grid spacing of $25 \mathrm{~km}$ (Ullrich et al., 2015). However, representation of the identified instabilities and mesovortices, starting to appear around $10 \mathrm{~km}$ grid spacing, exhibits physically different behavior from coarser-resolution simulations and consequentially violates the convergence assumption. Furthermore, should this phenomenon be of low predictability, as found for the simulations presented, it will inflate the uncertainty of a reference solution and thus deteriorate the explanatory power of the test. We thus motivate specifying a resolution range, including an upper and a lower bound, in the test protocol. However, the appropriate values may need to be determined empirically since no apparent scale separation has yet been identified for the atmosphere. Alternatively, employing bulk metrics could allow extending some established convergence-based tests to kilometer-scale resolution (Langhans et al., 2012; Panosetti et al., 2018).

Finally, the presented study is testimony to the usefulness of large computational meshes with kilometer-scale resolution for numerical weather prediction and case studies. Here we showcase that the representation of mesovortices emerging from barotropic instabilities requires an extensive computational domain such that the full extent of the respective parent cyclones is contained within the domain over the course of several days, which enables the simulation of the frontal scale collapse. The relevance of the phe-

\section{Acknowledgments}

We want to thank Tarun Chadha from the Centre for Climate Systems Modeling (C2SM) for the visualizations displayed in Figure 1 and Christian Zeman for preprocessing the boundary conditions obtained from the ECMWF archive. We want to acknowledge the Federal Office of Meteorology and Climatology MeteoSwiss, especially the team lead by Oliver Fuhrer, for their support and our fruitful collaboration. Furthermore, we thank Daniel Lüthi and Heini Wernli for insightful discussions and comments, and Ralph Rickli for making us aware of the spectacular cyclone of 23 February 2016. Additionally, we would like to thank two anonymous reviewers for their input and fruitful discussion. The Swiss National Science Foundation supported this work under Sinergia Grant CRSII2_154486/1 crCLIM and the Early Postdoc. Mobility Grant 178503 . Finally, we acknowledge the Partnership for Advanced Computing in Europe (PRACE) for awarding us compute resources to Piz Daint at the Swiss National Supercomputing Center (CSCS). The manuscript's primary data can be obtained from https://doi.org/ 10.3929/ethz-b-000373195. For the final version of the manuscript, it will be moved to the ETH E-collection and assigned a DOI. COSMO may be used for operational and for research applications by the members of the COSMO consortium. Moreover, within a license agreement, the COSMO model may be used for operational and research applications by other national (hydro)meteorological services, universities, and research institutes. The particular version of the COSMO model used in this study is based on the official version 5.0 with many additions to enable GPU capability and is available under a license (http:// www.cosmo-model.org/content/ consortium/licencing.htm). nomenon becomes apparent from cases of severe wind storms originating from atmospheric environments similar to those investigated in the current study (Fink et al., 2009).

\section{References}

Ban, N., Schmidli, J., \& Schär, C. (2014). Evaluation of the convection-resolving regional climate modeling approach in decade-long simulations. Journal of Geophysical Research: Atmospheres, 119, 7889-7907. https://doi.org/10.1002/2014JD021478

Belušić, A., Prtenjak, M. T., Güttler, I., Ban, N., Leutwyler, D., \& Schär, C. (2018). Near-surface wind variability over the broader Adriatic region: Insights from an ensemble of regional climate models. Climate Dynamics, 50(11), 4455-4480. https://doi.org/10.1007/ s00382-017-3885-5

Bhatia, H., Pascucci, V., \& Bremer, P. (2014). The natural Helmholtz-Hodge decomposition for open-boundary flow analysis. IEEE Transactions on Visualization and Computer Graphics, 20(11), 1566-1578. https://doi.org/10.1109/TVCG.2014.2312012

Bishop, C. H., \& Thorpe, A. J. (1994a). Frontal wave stability during moist deformation frontogenesis. Part II: The suppression of nonlinear wave development. Journal of the Atmospheric Sciences, 51(6), 874-888. https://doi.org/10.1175/1520-0469 (1994)051<0874:FWSDMD>2.0.CO;2

Bishop, C. H., \& Thorpe, A. J. (1994b). Frontal wave stability during moist deformation frontogenesis. Part I: Linear wave dynamics. Journal of the Atmospheric Sciences, 51(6), 852-873. https://doi.org/10.1175/1520-0469 (1994)051<0852:FWSDMD>2.0.CO;2

Bjerknes, J., \& Solberg, H. (1922). Life cycle of cyclones and the polar front theory of atmospheric circulation, vol. 3(1). Kristiania: Cammermeyers Bogh.

Chaboureau, J. P., \& Thorpe, A. J. (1999). Frontogenesis and the development of secondary wave cyclones in FASTEX. Quarterly Journal of the Royal Meteorological Society, 125(555), 925-940.

Clark, P., Roberts, N., Lean, H., Ballard, S. P., \& Charlton-Perez, C. (2016). Convection-permitting models: A step-change in rainfall forecasting. Meteorological Applications, 23(2), 165-181. https://doi.org/10.1002/met.1538

Dacre, H. F., \& Gray, S. L. (2006). Life-cycle simulations of shallow frontal waves and the impact of deformation strain. Quarterly Journal of the Royal Meteorological Society, 132(620), 2171-2190. https://doi.org/10.1256/qj.05.238

Dee, D. P., Uppala, S. M., Simmons, A. J., Berrisford, P., Poli, P., Kobayashi, S., et al. (2011). The ERA-Interim reanalysis: Configuration and performance of the data assimilation system. Quarterly Journal of the Royal Meteorological Society, 137(656), 553-597. https://doi. org/10.1002/qj.828

Edelmann, W. (1963). On the behaviour of disturbances in a baroclinic channel (Tech. Rep. No. 7). Frankfurt am Main, DE: DWD Research Department, Contract AF 61 (052)-373, Deutscher Wetterdienst. https://metlis.dwd.de/repository/DOC000001/B00225876.pdf

Fehlmann, R., \& Davies, H. C. (1999). Role of salient potential-vorticity elements in an event of frontal-wave cyclogenesis. Quarterly Journal of the Royal Meteorological Society, 125(557), 1801-1824. https://doi.org/10.1002/qj.49712555716

Fink, A. H., Brücher, T., Ermert, V., Krüger, A., \& Pinto, J. G. (2009). The European storm Kyrill in January 2007: Synoptic evolution, meteorological impacts and some considerations with respect to climate change. Natural Hazards and Earth System Sciences, 9(2), 405-423.

Förstner, J., \& Doms, G. (2004). Runge-Kutta time integration and high-order spatial discretization of advection-A new dynamical core for the LMK, COSMO Newsletter (Tech. Rep. no. 4). Frankfurt am Main, DE: Deutscher Wetterdienst. http://www.cosmomodel.org/ content/model/documentation/newsLetters/newsLetter04/chp9-6.pdf

Fu, G., Niino, H., Kimura, R., \& Kato, T. (2004). Multiple polar mesocyclones over the Japan Sea on 11 February 1997. Monthly Weather Review, 132(3), 793-814. https://doi.org/10.1175/1520-0493 (2004)132<0793:MPMOTJ>2.0.CO;2

Fuhrer, O., Chadha, T., Hoefler, T., Kwasniewski, G., Lapillonne, X., Leutwyler, D., et al. (2018). Near-global climate simulation at 1 km resolution: Establishing a performance baseline on 4888 GPUs with COSMO 5.0. Geoscientific Model Development, 11(4), 1665-1681. https://doi.org/10.5194/gmd-11-1665-2018

Hohenegger, C., Brockhaus, P., \& Schär, C. (2008). Towards climate simulations at cloud-resolving scales. Meteorologische Zeitschrift, 17(4), 383-394. https://doi.org/10.1127/0941-2948/2008/0303

Holton, J., \& Hakim, G. (2013). An introduction to dynamic meteorology. Amsterdam: Elsevier Science.

Hoskins, B. J., Draghici, I., \& Davies, H. C. (1978). A new look at the $\omega$-equation. Quarterly Journal of the Royal Meteorological Society, 104(439), 31-38. https://doi.org/10.1002/qj.49710443903 
Hoskins, B. J., \& West, N. V. (1979). Baroclinic waves and frontogenesis. Part II: Uniform potential vorticity jet flows-cold and warm fronts. Journal of the Atmospheric Sciences, 36(9), 1663-1680. https://doi.org/10.1175/1520-0469 (1979)036<1663:BWAFPI>2.0.CO;2

Imamovic, A., Schlemmer, L., \& Schär, C. (2017). Collective impacts of orography and soil moisture on the soil moisture precipitation feedback. Geophysical Research Letters, 44, 11,682-11,691. https://doi.org/10.1002/2017GL075657

Jablonowski, C., \& Williamson, D. L. (2006). A baroclinic instability test case for atmospheric model dynamical cores. Quarterly Journal of the Royal Meteorological Society, 132, 2943-2975.

Joly, A., Browning, K. A., Bessemoulin, P., Cammas, J. P., Caniaux, G., Chalon, J. P., et al. (1999). Overview of the field phase of the fronts and Atlantic Storm-Track EXperiment (FASTEX) project. Quarterly Journal of the Royal Meteorological Society, 125(561), 3131-3163. https://doi.org/10.1002/qj.49712556103

Joly, A., \& Thorpe, A. J. (1990). Frontal instability generated by tropospheric potential vorticity anomalies. Quarterly Journal of the Royal Meteorological Society, 116(493), 525-560. https://doi.org/10.1002/qj.49711649302

Keyser, D., Reeder, M. J., \& Reed, R. J. (1988). A generalization of Petterssen's frontogenesis function and its relation to the forcing of vertical motion. Monthly Weather Review, 116(3), 762-781. https://doi.org/10.1175/1520-0493 (1988)116<0762:AGOPFF>2.0.CO;2

Langhans, W., Schmidli, J., \& Schär, C. (2012). Bulk convergence of cloud-resolving simulations of moist convection over complex terrain. Journal of the Atmospheric Sciences, 69(7), 2207-2228.

Leutwyler, D., Fuhrer, O., Lapillonne, X., Lüthi, D., \& Schär, C. (2016). Towards European-scale convection-resolving climate simulations with GPUs: A study with COSMO 4.19. Geoscientific Model Development, 9(9), 3393-3412. https://doi.org/10.5194/gmd-9-3393-2016

Ludwig, P., Pinto, J. G., Hoepp, S. A., Fink, A. H., \& Gray, S. L. (2015). Secondary cyclogenesis along an occluded front leading to damaging wind gusts: Windstorm Kyrill, January 2007. Monthly Weather Review, 143(4), 1417-1437. https://doi.org/10.1175/MWR-D-14-00304.1

Malardel, S., Joly, A., Courbet, F., \& Courtier, P. H. (1993). Nonlinear evolution of ordinary frontal waves induced by low-level potential vorticity anomalies. Quarterly Journal of the Royal Meteorological Society, 119(512), 681-713. https://doi.org/10.1002/qi.49711951205

Moore, G. W. K., \& Peltier, W. R. (1987). Cyclogenesis in frontal zones. Journal of the Atmospheric Sciences, 44(2), 384-409. https://doi.org/10.1175/1520-0469 (1987)044<0384:CIFZ>2.0.CO;2

Müller, S. K., Manzini, E., Giorgetta, M., Sato, K., \& Nasuno, T. (2018). Convectively generated gravity waves in high resolution models of tropical dynamics. Journal of Advances in Modeling Earth Systems, 10, 2564-2588. https://doi.org/10.1029/2018MS001390

Panosetti, D., Schlemmer, L., \& Schär, C. (2018). Convergence behavior of idealized convection-resolving simulations of summertime deep moist convection over land. Climate Dynamics. https://doi.org/10.1007/s00382-018-4229-9

Park, S., Skamarock, W. C., Klemp, J. B., Fowler, L. D., \& Duda, M. G. (2013). Evaluation of global atmospheric solvers using extensions of the Jablonowski and Williamson baroclinic wave test case. Monthly Weather Review, 141(9), 3116-3129.

Prein, A. F., Langhans, W., Fosser, G., Ferrone, A., Ban, N., Goergen, K., et al. (2015). A review on regional convection-permitting climate modeling: Demonstrations, prospects, and challenges. Reviews of Geophysics, 53, 323-361. https://doi.org/10.1002/2014RG000475

Ralph, M. F. (1996). Observations of 250-km-wavelength clear-air eddies and 750-km-wavelength mesocyclones associated with a synoptic-scale midlatitude cyclone. Monthly Weather Review, 124(6), 1199-1210. https://doi.org/10.1175/1520-0493(1996)124 $<1199:$ OOKWCA $>2.0$. CO;2

Reinhardt, T., \& Seifert, A. (2006). A three-category ice-scheme for the LMK (Tech. Rep. No. 6): COSMO. Model Development and Application, COSMO Newsletter. http://www.cosmo-model.org/content/model/documentation/newsLetters/newsLetter06/cnl6_ reinhardt.pdf

Rivals, H., Cammas, J. P., \& Renfrew, I. A. (1998). Secondary cyclogenesis: The initiation phase of a frontal wave observed over the eastern Atlantic. Quarterly Journal of the Royal Meteorological Society, 124(545), 243-267. https://doi.org/10.1002/qi.49712454511

Schär, C., \& Davies, H. C. (1990). An instability of mature cold fronts. Journal of the Atmospheric Sciences, 47(8), 929-950. https://doi.org/10.1175/1520-0469 (1990)047<0929:AIOMCF>2.0.CO;2

Schär, C., \& Wernli, H. (1993). Structure and evolution of an isolated semi-geostrophic cyclone. Quarterly Journal of the Royal Meteorological Society, 119(509), 57-90. https://doi.org/10.1002/qj.49711950904

Schemm, S., \& Sprenger, M. (2015). Frontal-wave cyclogenesis in the North Atlantic—A climatological characterisation. Quarterly Journal of the Royal Meteorological Society, 141(693), 2989-3005. https://doi.org/10.1002/qj.2584

Shutts, G. J., \& Vosper, S. B. (2001). Stratospheric gravity waves revealed in NWP model forecasts. Quarterly Journal of the Royal Meteorological Society, 137(655), 303-317. https://doi.org/10.1002/qj.763

Snyder, C. (1995). Stability of steady fronts with uniform potential vorticity. Journal of the Atmospheric Sciences, 52(6), 724-736. https://doi.org/10.1175/1520-0469 (1995)052<0724:SOSFWU>2.0.CO;2

Steppeler, J., Doms, G., Schättler, U., Bitzer, H. W., Gassmann, A., Damrath, U., \& Gregoric, G. (2003). Meso-gamma scale forecasts using the nonhydrostatic model LM. Meteorology and Atmospheric Physics, 82(1-4), 75-96.

Ullrich, P. A., Reed, K. A., \& Jablonowski, C. (2015). Analytical initial conditions and an analysis of baroclinic instability waves in $f$ and betaplane 3D channel models. Quarterly Journal of the Royal Meteorological Society, 141(693), 2972-2988. https://doi.org/10.1002/qj.2583

Vosper, S. B., Brown, A. R., \& Webster, S. (2016). Orographic drag on islands in the NWP mountain grey zone. Quarterly Journal of the Royal Meteorological Society, 142(701), 3128-3137. https://doi.org/10.1002/qj.2894 